\title{
Pectoralis Major Flap: Interest in Maxillofacial Reconstruction About A Case
}

\author{
N'diaye A*, Khalfi L, Chabi W, Sabani H, El Khatib K \\ Departement of Plastic and Maxillo-facial Surgery/ 'Mohammed V' Teaching Armed Forces Hospital 10100 Rabat Morocco
}

\begin{tabular}{ll}
\hline DOI: $10.36348 /$ SJMPS.2019.v05i10.007 & | Received: 21.09.2019| Accepted: 28.09.2019 | Published: 25.10 .2019 \\
*Corresponding author: Ndiaye &
\end{tabular}

Abstract

The pectoralis major myocutaneous flap (PMMC) was first described by Pickrell in 1947, and perfected in 1979 by Ariyan. The reconstruction of oral cavity defects represent a challenge esthetically and functionally. The case reported, we reconstructed oral mucosa and skin cheek-labial in one-step procedure preserving the deltopectoral flap from the same side. The patient was a 55-year-old male who had sustained squamous cell carcinoma. The tumor ablation resulted in oral cavity exposure. A PMMC flap was used to reconstruct oral mucosa and skin cheek. We opted to preserve the deltopectoral flap in the event that skin island necrosis did occur. The pedicle pectoralis major myocutaneous (PMMC) flap based on the thoracoacromial artery procedure, has been widely used in the reconstruction of head and neck defects but in literature a few case of oral reconstruction is reported. PMMC flap applications mainly in oral cancer surgery include the tongue, floor of mouth, buccal, mandible reconstruction, reconstruction of full thickness defects of the cheek. It's due to its simple technical aspects, versatility and proximity to the oral cavity region. Disadvantages of the PMMC are reported and minor complications that did not require second procedures. The use of PMMC flaps in reconstructive oral surgery is a safe, quick, one-step procedure, not requiring microvascular experience that is particularly indicated in elderly patients and/or those with severe medical comorbidities. Whether the low rate of complications and donor site morbidity, we consider that the well-being of the patient should not be underestimated.

Keyswords: Oral cancer, squamous cell carcinoma, pedicled flaps, pectoralis major myocutaneous flap, deltopectoral flap, oral reconstruction, cheek, labial, surgical procedure. Oral cancer, squamous cell carcinoma, pedicled flaps, pectoralis major myocutaneous flap, deltopectoral flap, oral reconstruction, cheek, labial, surgical procedure.

Copyright @ 2019: This is an open-access article distributed under the terms of the Creative Commons Attribution license which permits unrestricted use, distribution, and reproduction in any medium for non-commercial use (NonCommercial, or CC-BY-NC) provided the original author and source are credited.

\section{INTRODUCTION}

The pectoralis major myocutaneous flap (PMMC) was first described by Pickrell in 1947[1], and perfected by in 1979 by Ariyan for the reconstructive methods in head and neck defects. The reconstruction oral cavity defects represent a challenge because of the critical role of this area both esthetically and functionally. A cutaneous paddle can be used to reconstruct oral mucosa, skin, or both. Despite the recent advances in free flap surgery, the pedicle PMMC flap maintains a role in head and neck reconstruction, especially in the oral cavity. The PMMC is characterized by a simple procedure and a short time to harvest. The case we presented here is interesting of its management in major oral cancer surgery but also to reconstruct oral mucosa and skin cheek in one-step procedure preserving the deltopectoral flap from the same side in case of secondary procedure reconstruction

\section{OBSERVATION}

The patient was a 55-year-old male who had sustained squamous cell carcinoma in internal side of the right cheek extended to the labial commissure area (Figure 1, 2).

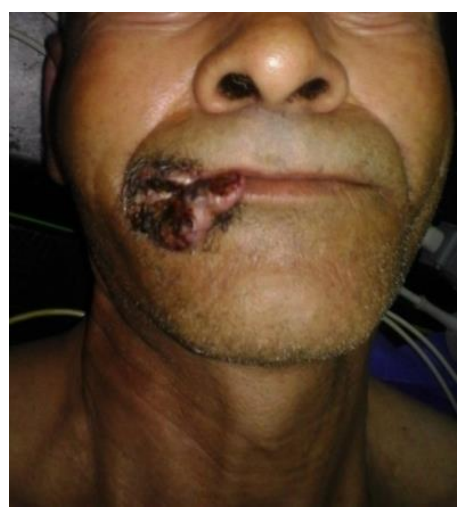

Fig-1: Patient 55 years-old with carcinoma in internal side of the right cheek extended to the labial commissure area 


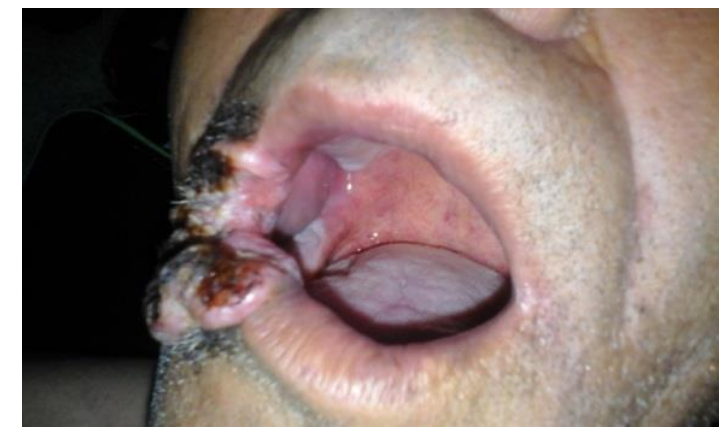

Fig-2: Oral view carcinoma in internal side of the right cheek

He also had a history of tobacco addiction. He was transferred to our care hospital and received an ablation surgery followed by a radical neck dissection as primary operation. The tumor ablation resulted in labial major skin defect and oral cavity exposure (figures $3,4,5$ )

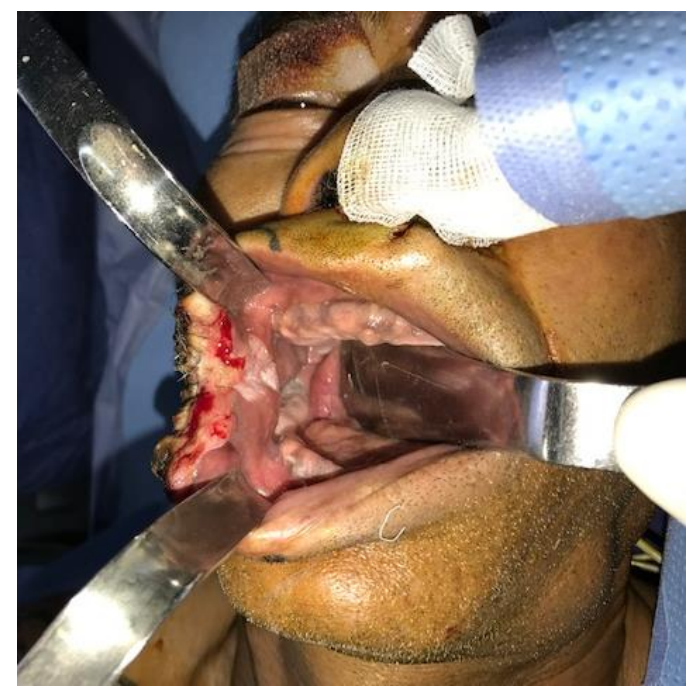

Fig-3: Operative view carcinoma in internal side of the right cheek

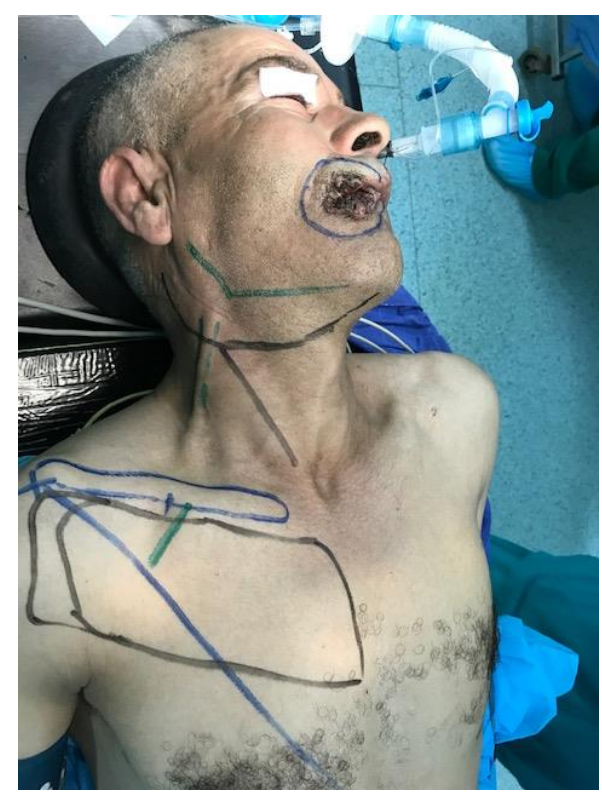

Fig-4: operative view conventional design based on a PMMC flap

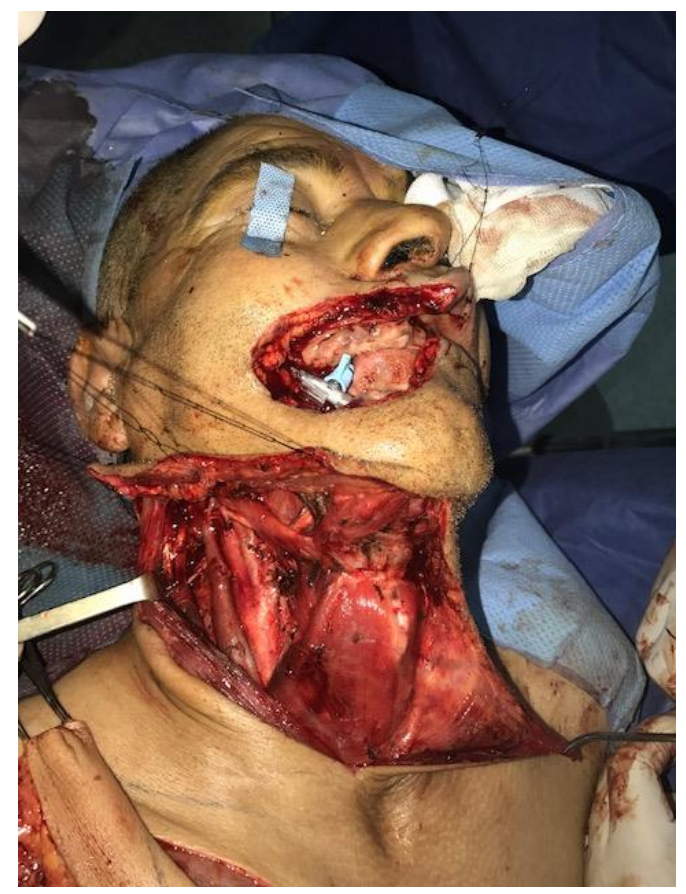

Fig-5: Oral defect after tumor resection and neck dissection

For oral defects after cancer resection we chose a pedicle pectoralis major myocutaneous (PMMC) flap for immediate reconstruction because of the age of the patient and the presence of comorbidity. A cutaneous paddle was used to reconstruct cheek and skin commissure of the lips. We decide to preserve the deltopectoral flap in the event that skin island necrosis did occur and would have the possibility of harvesting the deltopectoral to cover the pectoralis muscle (figures $6,7,8,9)$.

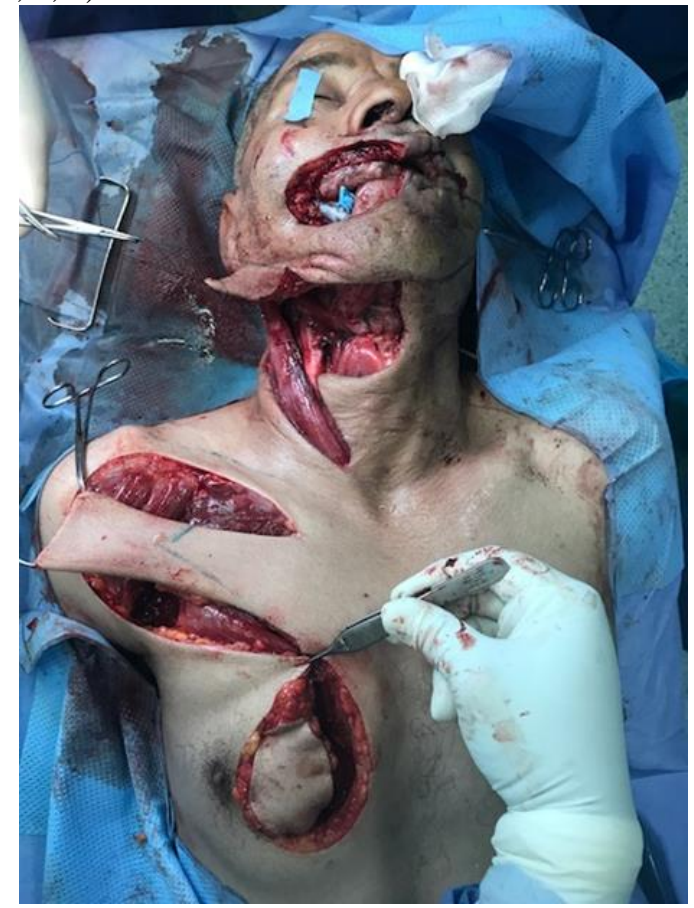

Fig-6: Possibility of harvesting a deltopectoral and pectoralis major myocutaneous flap with his skin paddle from the same side 


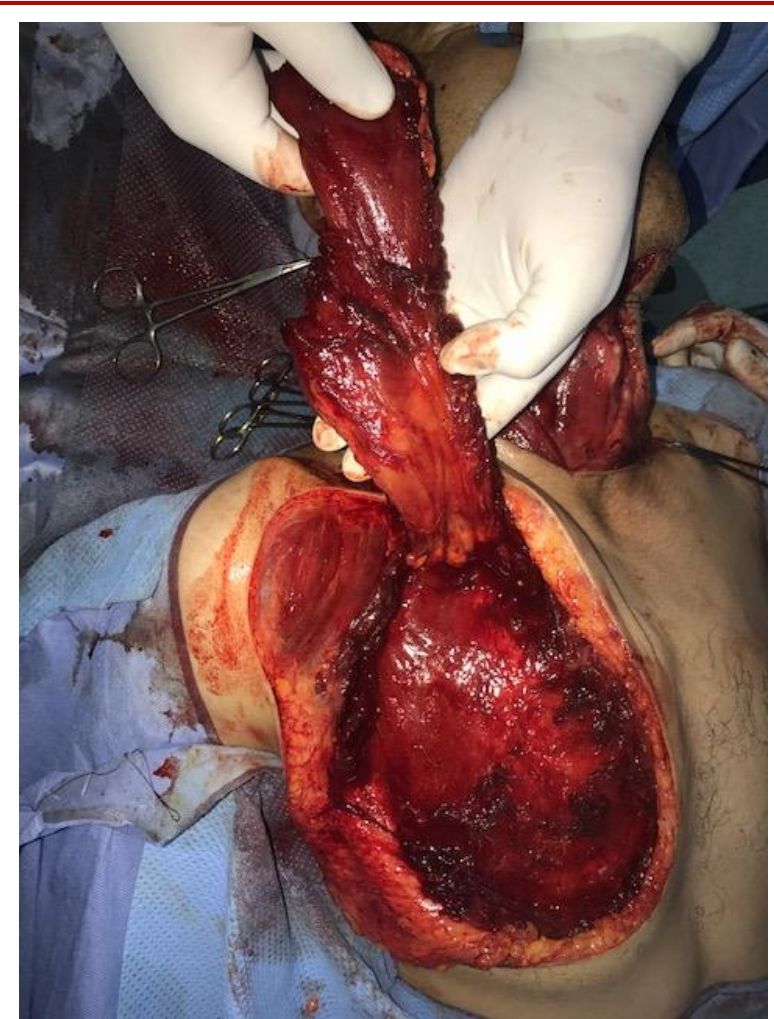

Fig-7: Operative view pectoralis major flap with his skin paddle was elevated. The pectoral branch of the thoracoacromial artery was identified beneath the pectoralis major

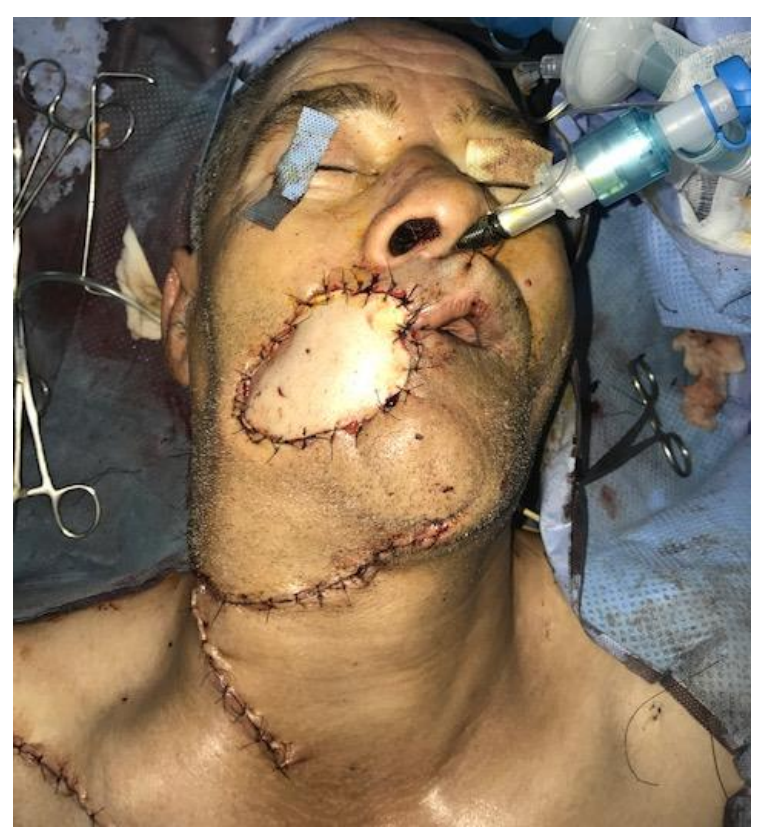

Fig-8: The large oral defect was closed with a PMMC flap with a large skin paddle after the flap is being passed underneath the skin bridge

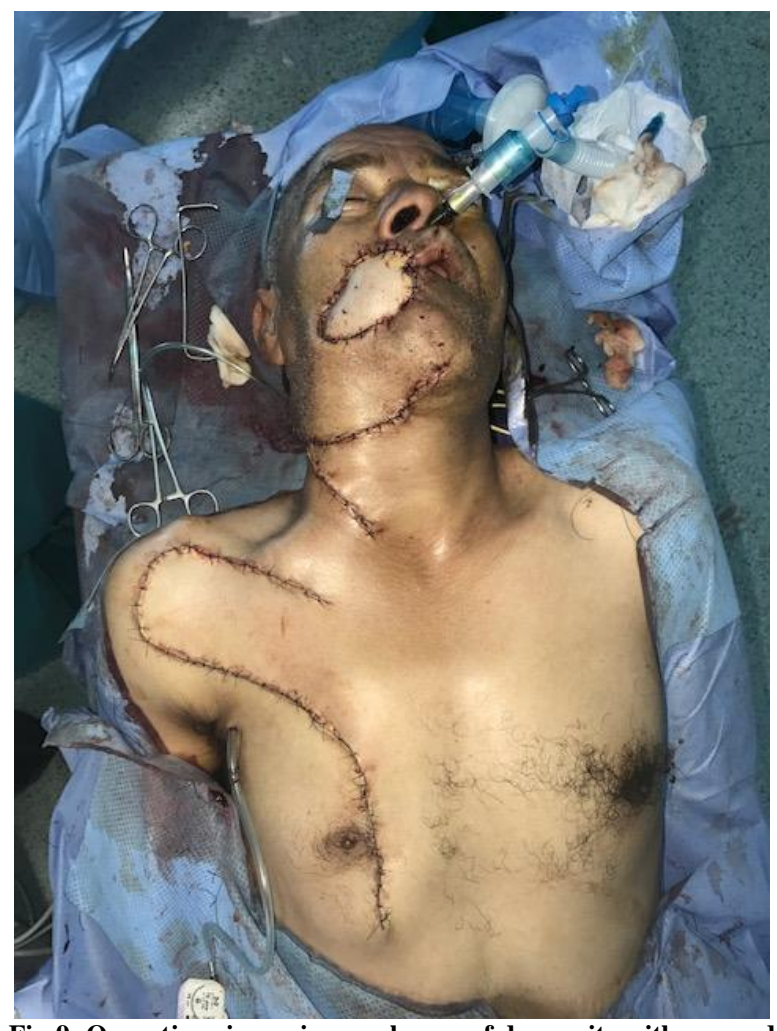

Fig-9: Operative view primary closure of donor site with no need for a skin graft.

The postoperative follow-up was uneventful with complete survival of the flap (Figures 10, 11, 12, 13).

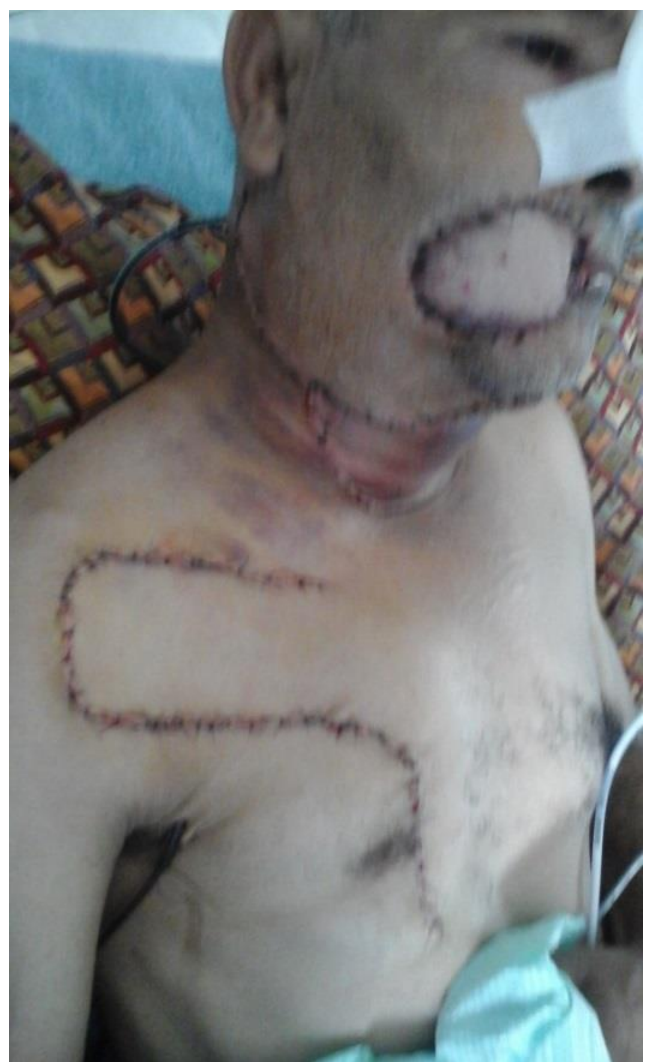

Fig-10: Postoperative care view at 48 Hours follow-up 


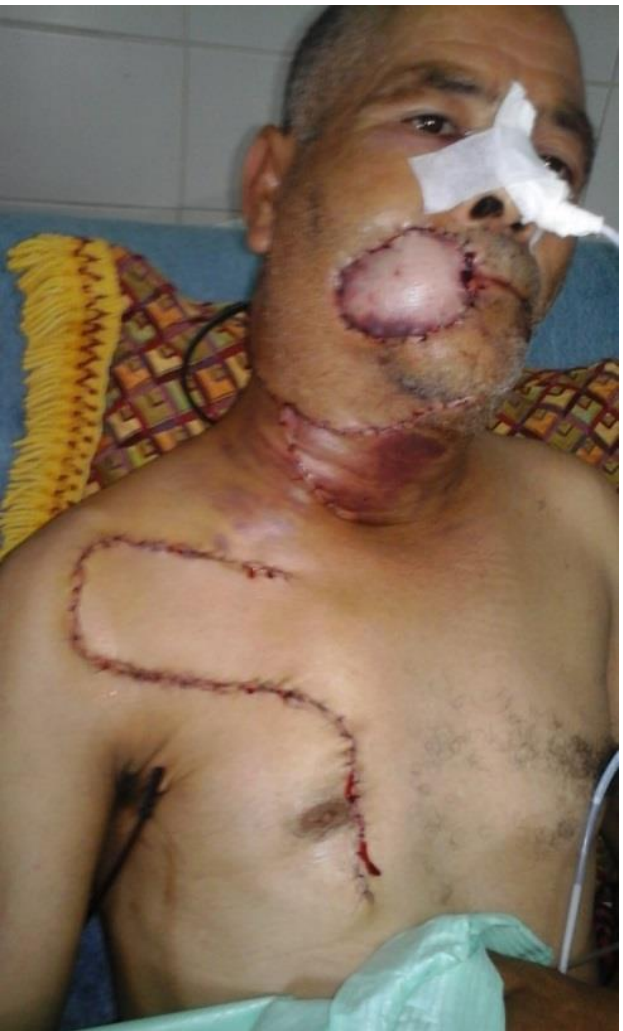

Fig-11: Postoperative care view the donor site a few days after intervention

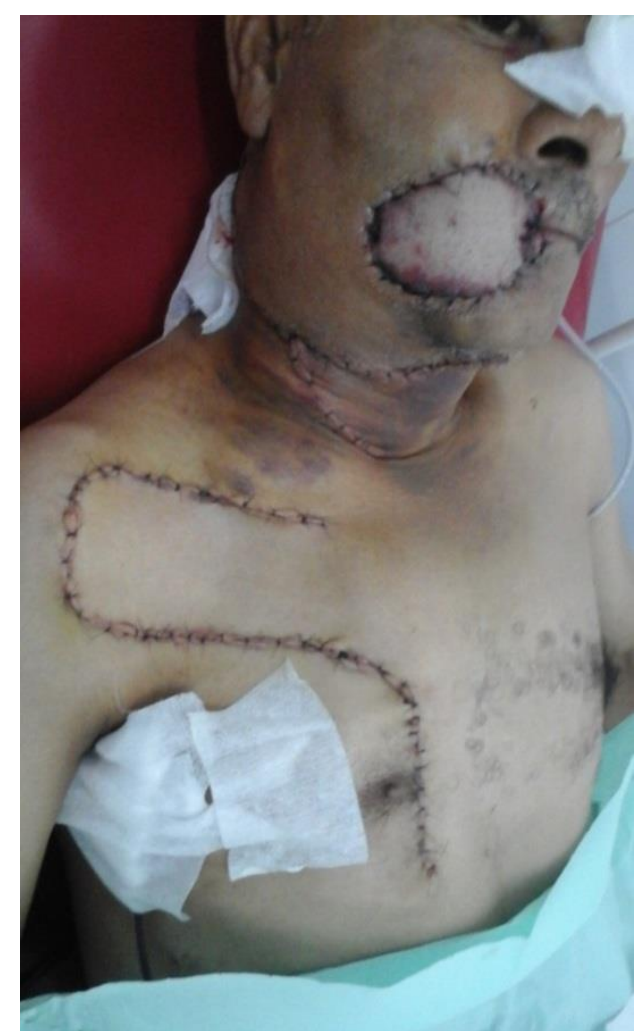

Fig-12: the donor site was successfully performed without any complication

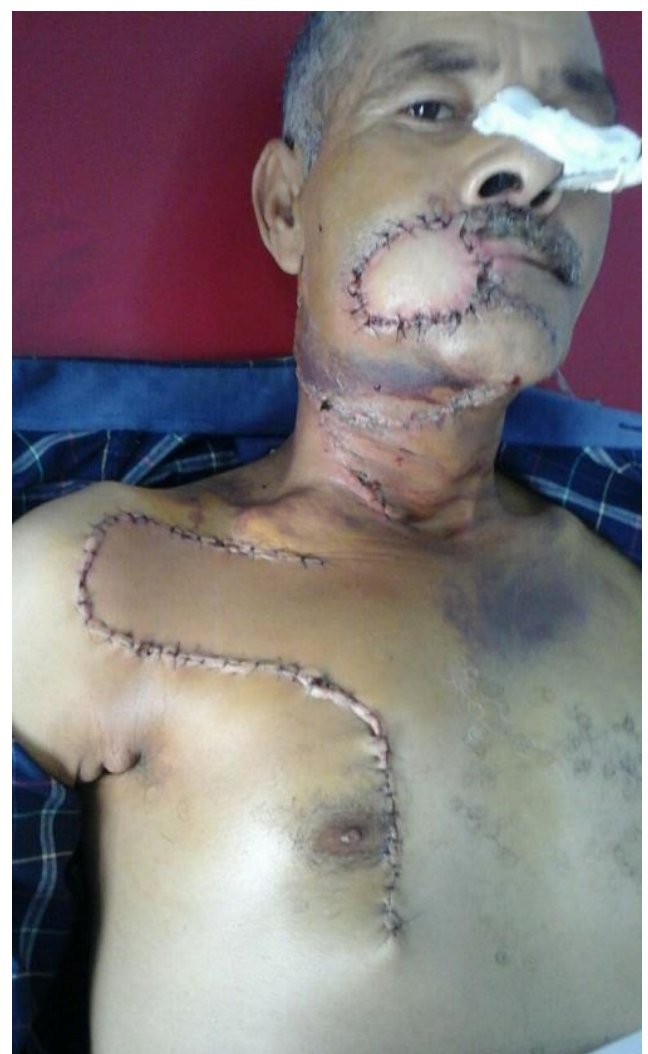

Fig-13: control two weeks after surgery, preserving the deltopectoral flap from the same side in case of secondary reconstruction

The patient received postoperative adjuvant radiotherapy at the right neck .No delayed wound disruption or flap necrosis was noted 6 months after surgery .The goal of reconstruction is a safe, one-stage procedure that allows early functional recovery and adequate ability to tolerate postoperative radiation.

\section{DISCUSSION}

The pectoralis major muscle flap was first described by Pickrell in 1947 as a turnover flap in chest wall reconstruction [1] but the pedicle pectoralis major myocutaneous (PMMC) flap based on the thoracoacromial artery procedure was originally developed by Dr Ariyan in 1979 and has been widely used in the reconstruction of head and neck defects [25].On human anatomy the pectoralis major has a clavicular head, a sternocostal head, and an abdominal portion $[1,6]$. It was shown that the skin obtains its blood supply through the underlying muscle [7]. Since then, an impressive number of modifications to the original technique have been proposed over the years [2]. In general, the pectoralis major myofascial (PMMF) and PMMC, are by far the most employed techniques [2]. The PMMF is consisting in the use of pectoralis major muscle alone with overlying fascia without the skin paddle [4]. While PMMC is more suitable for reconstruction involving skin defects, the PMMF variant better complies with mucosal defects [2]. This is a commonly used flap in reconstructive head and neck surgery $[4,8]$ but in literature a few case 
of oral reconstruction is reported. In our case the patient, with oro-labial squamous cell carcinoma was treated by tumor ablation and neck dissection with the need of primary reconstruction.

The reconstruction oral cavity defects represent a challenge because of the critical role of this area both esthetically and functionally [5]. PMMC flap applications mainly in oral surgery include the tong, floor of mouth, followed by buccal, mandible reconstruction when a free flap is not advisable by rolling the PMMC on a metal plate, reconstruction of full thickness defects of the cheek $[2,5]$. In this case a cutaneous paddle is used to reconstruct both tissues Cheek and skin of the labial commissure. PMMC is well established as one of the most of the important reconstructive methods in general oral cancer surgery due to its simple technical aspects, versatility, and proximity to the oral cavity region [5]. The PMMC is well established as one of the most important reconstructive methods in major oral cancer surgery due to its simple technical aspects, versatility and proximity to the oral cavity region [5]. The PMMC is now popularizes in developing countries with limited medical resources. Whereas it is used much less in western countries where availability of microsurgical techniques is more widespread [9]. As free flap the anterolateral thigh perforator free flaps (ALTFF) was first reported by song in 1984 in oral cavity reconstructions after major oral cancer surgery [5]. The superiority and benefits of free flaps for repairing head and neck defects have been well described [10,11], the use of a pedicled flaps note only represents an alternative to free tissue transfert, but is also a preferable option, with fewer risks for the patient [11] as poor vascular status, previous radiation $>60$ Gy to the neck, poorly controlled diabetes, systemic vascular sclerosis, compromised general status, ASA grade 3-4, âge $>75$, major vessel protection, vessel depleted neck, total glossectomy, salvage reconstruction, flap failure, fistula [9]. We have proposed to the patient a pedicle PMMC flap for reconstruction because his age the comorbidity as tobacco addition, no smoking cessation yet, oral reconstruction and the previous adjuvant radiotherapy. The advantages of this procedure compary with a free flap as the shorter operation time, less donor site morbidity, and less need for the postoperative intensive care -monitoring [3]. The PMMC flap is technically easier and much faster to harvest, and does not require microvascular experience [3]. Before harvesting, we considered the possibility of partial or total skin paddle necrosis of the PMMC flap. In the event that skin island necrosis did occur, we would have the possibility of harvesting a deltopectoral flap to cover the pectoralis muscle. Few studies have evaluated the differences in quality of life between patients with oral cavity cancers reconstructed with PMMC compared with those who underwent free flap [5]. Disadvantages of the PMMC include the need to rotate the vascular pedicle of the flap $180^{\circ}$ when using the skin paddle, a reduced neck mobility, inconsistent blood circulation, deformity of the thoracic wall, function impairment of the neck and shoulder, as hair growth, excessive bulk in some situations, and possibly poor function outcome of the recipient site [8, 9]. Mehrhof et al. reported a complication rate of $54 \%$ with major complications were total flap necrosis (4-7\%) and partial flap necrosis (12-27\%). The vast majority were minor complications that didn't require second procedures for correction: orocutaneous fistulas (16, $5 \%$ ), suture line dehiscence (12\%), neck wound complications $(11 \%)$, donor sire complication $(1,3 \%)$ $[2,4]$. In addition post-operative care, patient was instructed to limit range of motion exercises to minimize harvested muscle movement. No delayed wound disruption of flap necrosis was noted 6 month after surgery. The patient presented pain, bruise, swallowing, and anxiety and salvia incontinence overs the day following the surgery. Chewing, speech and shoulder domains were considered the least important to patient. We have seen that the donor site scar affected the patients, normal social activities and social interaction, so it brings a great deal of distress to him.

\section{CONCLUSION}

The use of PMMC flaps in reconstructive oral surgery is a safe, quick, one-step procedure, not requiring microvascular experience that is particularly indicated in elderly patients and/or those with severe medical comorbidities. Another possibility is the harvesting of a deltopectoral and pectoralis major flap from the same side in case of secondary procedure reconstruction. Whether the low rate of complications and donor site morbidity, we consider that the wellbeing of the patient should not be underestimated.

\section{ACKNOWLEDGEMENT}

The authors declare that there is no conflict of interest

\section{REFERENCES}

1. Samir Mardini, M.D. (2011) Chest Wall Reconstruction; Guest Editors, Semin Plast Surg; 25:43-54.

2. Bussu, F., Gallus, R., Navach, V., Bruschini, R., Tagliabue, M., Almadori, G., ... \& Calabrese, L. (2014). Contemporary role of pectoralis major regional flaps in head and neck surgery. Acta Otorhinolaryngologica Italica, 34(5), 327.

3. Montemari, G., Rocco, A., Galla, S., Damiani, V., \& Bellocchi, G. (2012). Hypopharynx reconstruction with pectoralis major myofascial flap: our experience in 45 cases. Acta Otorhinolaryngologica Italica, 32(2), 93.

4. Huang, J. W., Wu, N. Y., \& Lin, Y. Y. (2017). Using a pedicle pectoralis major musculocutaneous flap in head and neck reconstruction after modified 
radical mastectomy: A case report. Medicine, 96(15).

5. Xiao, Y., Zhu, J., Cai, X., Wang, J., Liu, F., \& Wang, H. (2013). Comparison between anterolateral thigh perforator free flaps and pectoralis major pedicled flap for reconstruction in oral cancer patients-A quality of life analysis. Medicina oral, patologia oral y cirugia bucal, 18(6), e856.

6. Potau, J. M., Arias-Martorell, J., BelloHellegouarch, G., Casado, A., Pastor, J. F., de Paz, F., \& Diogo, R. (2018). Inter-and Intraspecific Variations in the Pectoral Muscles of Common Chimpanzees (Pan troglodytes), Bonobos (Pan paniscus), and Humans (Homo sapiens). BioMed research international, 2018.

7. Kim, J. T., \& Kim, S. W. (2015). Perforator flap versus conventional flap. Journal of Korean Medical Science, 30(5), 514-522.
8. Kruse, A. L., Luebbers, H. T., Obwegeser, J. A., Bredell, M., \& Grätz, K. W. (2011). Evaluation of the pectoralis major flap for reconstructive head and neck surgery. Head \& neck oncology, 3(1), 12.

9. Liu, M., Liu, W., Yang, X., Guo, H., \& Peng, H. (2017). Pectoralis major myocutaneous flap for head and neck defects in the era of free flaps: harvesting technique and indications. Scientific reports, 7, 46256.

10. Wei, W., Qiu, Y., Fang, Q., \& Jia, Y. (2019). Pectoralis major myocutaneous flap in salvage reconstruction following free flap failure in head and neck cancer surgery. Journal of International Medical Research, 47(1), 76-83.

11. Salvatori, P., Motto, E., Paradisi, S., Zani, A., Podrecca, S., \& Molinari, R. (2007). Oromandibular reconstruction using titanium plate and pectoralis major myocutaneous flap. Acta Otorhinolaryngologica Italica, 27(5), 227. 